\title{
PATIENTS ON THE ROLE OF MODERN MIDWIVES IN CONDUCTING A NORMAL BIRTH
}

\author{
Kremena Miteva, Zdravka Atanasova \\ Department of Health Care, Medical Faculty, Trakia UniversityDepartment of Health Care, \\ Faculty of Public Health, Medical University of Varna
}

\begin{abstract}
INTRODUCTION: Midwifery care is an essential factor on which the optimum completion of labor depends. Therefore, the correlation between the quality of the professional activities of midwives in the delivery room and the level of satisfaction of patients is of great importance.

AIM: This scientific study is conducted in order to explore and analyze the opinion of patients on the role of the modern midwife during normal birth.

MATERIALS AND METHODS: The study includes 76 patients /women who have given birth/ from the University Hospital for Active Treatment "Stoyan Kirkovich" - Stara Zagora in the period January-June 2016. A criterion for inclusion in the study was a definitive diagnosis - vaginal delivery. A sociological method was used anonymous questionnaire. The data were processed with the statistical package SPSS.

RESULTS AND CONCLUSION: The results allowed us to draw the conclusion that there is a tendency to neglect the integrated approach in the implementation of obstetric care at the expense of instrumental care. Also, patients define the work of the midwife as a significant factor for the optimal flow of the process of birth.
\end{abstract}

Keywords: midwife, labor, patients, satisfaction

\section{INTRODUCTION}

The midwife is a part of the medical team during one of the most important moments for each woman - the birth. Obstetric care is a significant factor, on which its optimal results depend. Present reality, however, registers some negative trends - shortage and turnover of obstetric staff, lowered work motivation of the health care professionals and diminished prestige of the Bulgarian midwife. Simultaneously, the requirements of patients and employers re-

\footnotetext{
Address for correspondence:

Kremena Miteva

Department of Health Care,

Medical Faculty

Trakia University

11 Armeyska St.

Stara Zagora

e-mail:kremena.miteva@abv.bg
}

garding the quality of health services are growing. Increasingly discussed among the guild is the problem of the limited competence of midwives in our country compared to their counterparts in the other EU countries.

These trends focus on the correlation between the quality of the professional activities of the midwives in the delivery room and the level of satisfaction of the patients, as well as raise questions about the role of the Bulgarian midwife in the system of maternal health.

Bulgarian legislation (Regulation №19 on approving the medical standard "Obstetrics and Gynecology", Regulation №1 on professional activities that midwives may perform on instruction or by themselves) regulates the activities of the midwife in assisting in a normal birth, namely $(2,3)$ : 
$\diamond$ preparation of the delivery room /instruments, medicines and necessary equipment to conduct normal birth/;

$\diamond$ auscultation of children's heart tones/beats;

$\diamond$ performance of a cardiotocography record;

$\diamond$ measuring and recording the pelvic diameters of the pregnant woman;

$\diamond$ external obstetric examination;

$\diamond$ monitoring the general condition of the labor and interpretarion of the results;

$\diamond$ preparation and administration of oxytocin infusion for stimulation/induction of labor;

$\diamond$ placing of a permanent urethral catheter;

$\diamond$ preparation and care of the woman in labor in case of an instrumental break of the amniotic sac;

$\diamond$ monitoring and manipulation of the woman in labor in the stage of cervical dilation;

$\diamond$ assisting in the second stage of birth - cephalic presentation of fetus;

$\diamond$ assisting in the third stage of birth;

$\diamond$ monitoring and care for the woman in labor in the early post placental period and diagnosing problems requiring medical intervention;

$\diamond$ first care for the newborn child;

$\diamond$ preparation of the documentation of the woman in labor;

$\diamond$ preparation of the documentation of the newborn;

$\diamond$ preparing the necessary instruments in case of surgical interventions for expansion and restoration of soft birth canals;

$\diamond$ performing episiotomy in the presence of a doctor.

The quality of the technological part of the process of providing obstetric care during labor is essential, but not the only factor for its optimal flow. Crucial for each patient is the birth of the child to be a positive experience. It is therefore essential for each midwife to focus on the care for the mother and the child and to work mainly in their own interest, without ignoring the interests of the hospital and the health system.
AIM

This scientific report aims at exploring and analyzing the opinion of patients on the role of the modern midwife in conducting a normal birth.

\section{MATERIALS AND METHODS}

The survey was conducted in connection with the implementation of a research project №15/2015 of the Trakia University - "Update of Training on Practical Foundations of Nursing and Midwifery Care with Students from the Midwife Program". The study included 76 patients /women who have given birth/ from the University Hospital for Active Treatment "Stoyan Kirkovich" - Stara Zagora during the period January - June 2016. The criterion for inclusion in the study was a definitive diagnosis - normally proceeded birth. A sociological method was used - anonymous questionnaire containing 11 questions of a closed-ended type. The results were processed with the program SPSS for mathematical and statistical analysis.

\section{RESULTS}

The largest share of respondents was in the age group $20-24$ years - $34.21 \%$, followed by those between $25-29$ years $-21.05 \%$, to 19 years $-17.11 \%$ and $30-34-14.47 \%$. The proportion of patients over 35 years $-13.16 \%$ was the lowest.

The educational level of the respondents was as follows: the survey was dominated by the groups of respondents with secondary education and higher education (respectively $43.42 \%$ and $38.16 \%$ ). The proportion of those with primary education was $18.42 \%$.

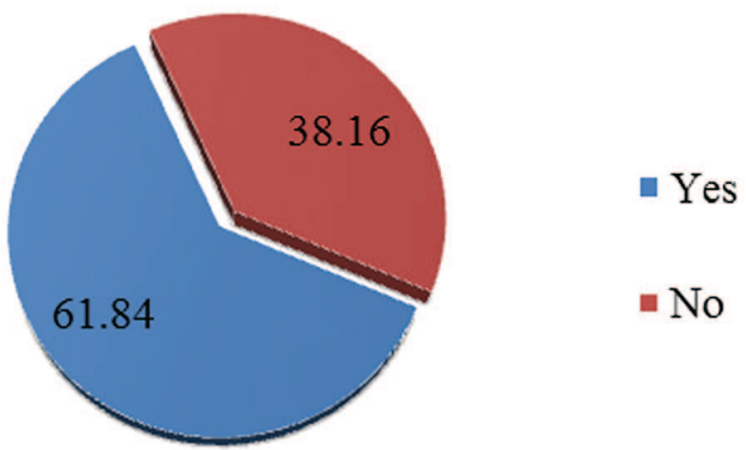

Figure 1. Awareness of the patients of the midwife assisting in the delivery 
The significance of the midwife as part of the team in conducting a normal birth derives from its regulated core functions. Interest in the study present respondents' answers to the questions "Do you know the name of the midwife who assisted in your delivery?" (Fig. 1):

A significant proportion $(n=47)$ of the mothers know the name of their midwife. Approximately the same number $(n=49)$ know the name of the doctor. However, a relatively high percentage of the patients (nearly 40\%) do not know the names of the team (Fig. 1). The reasons for this can be found in gaps in communication between the midwife and the woman in labor or in the lack of interest of the patients.

Conducting a normal birth involves continuous active surveillance by the midwife. Significant amount of time for care is spent during the first stage in the prenatal room. This leads to the building of the foundations of trust between the midwife and the patient, who wants to see and feel understanding, respect and support of her emotional experiences such as anxiety, doubts and mostly competent medical aid. This is related to the respondents' assessment of whether the midwife has spent enough time of observation and care during the expansion of the cervix (Fig. 2).

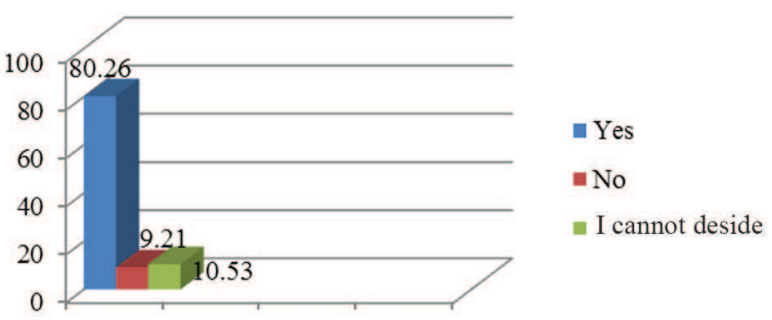

Figure 2. Satisfaction of the patients by the time spent by the midwife for monitoring and care in the prenatal hall

Figure 2 displays the high proportion of mothers $-80.26 \%(n=61)$ saying that the midwife, who assisted in their delivery, has devoted sufficient amount of time and attention in the first stage.

Empathy, understanding the emotional state of nonverbal signals of the patient, is a key skill that the midwives should manifest in their work. The ability to understand the experiences of the other person, the ability of emotional empathy, compassion, sharing the feelings and emotional state of the other, is es- sential for the achievement and maintenance of good relationships with patients, as well as for optimization of the overall process of providing obstetric care (1).

The research found that according to the half of the studied contingent $(n=38)$, midwives ignore the integrated approach in the implementation of health care at the expense of instrumental care. According to the other half, the midwife has provided high enough level of psycho-emotional support during the labor (Fig. 3):

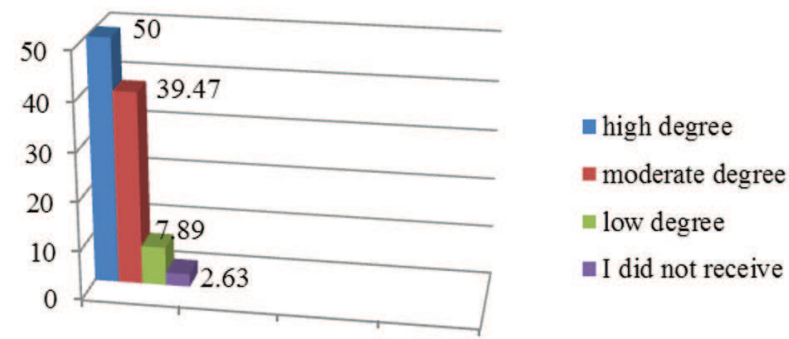

Figure 3. Degree of psycho-emotional support, provided by the midwife during childbirth, according to the patients

The verbal communication midwife-patient is a key factor influencing the optimal course of the second stage of birth - the expulsion of the fetus. Preventing complications requires prior skillful, clear, comprehensible and accessible instruction, consistent with her social, educational and emotional status.

The study found that a high proportion of mothers $(n=56)$ were fully satisfied by the way the instruction was held by the midwife. Partially satisfied, according to this indicator, were nearly $20 \%$ of the respondents, and unsatisfied were 6.58\% (Fig. 4). Similar is the assessment of the patients of the indi-

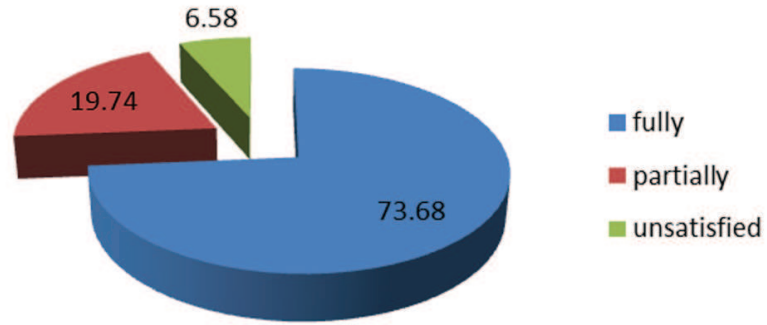

Figure 4. Satisfaction of mothers by instructions of the midwife for a second period of birth 
cators of satisfaction of the behavior and attitude of the midwife during the birth of the fetus and the early postplacental stage.

The current Bulgarian system of maternity care does not regulate the right to choose a midwife in assisting in the birth. In order to establish the significance of the work of the midwife, of great interest for the study are the responses of mothers to the question: "If you had a choice, would you choose the midwife to assist in your delivery?" (Fig. 5):

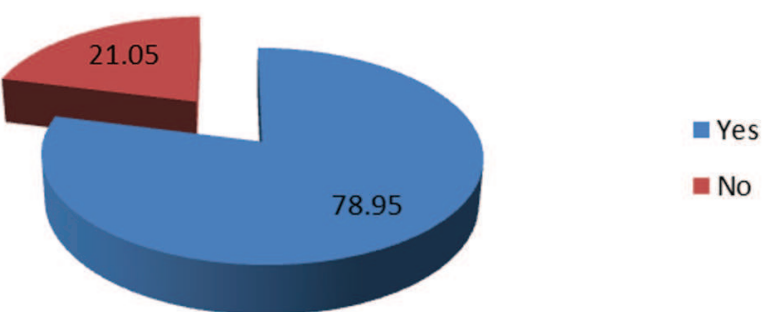

Figure 5. Patients about the possibility of free choice of a midwife during childbirth

The data in Figure 5 confirm the appreciation of the importance of midwifery care in conducting a normal birth and recognition of the work of the midwife - almost $80 \%$ of mothers $(n=60)$ prefer to be able to choose the midwife.

\section{CONCLUSION}

This study confirmed the important role of the modern midwife in assisting in a normal birth. The results give the possibility to formulate the following conclusions:

$\diamond$ The patients determine the work of the Bulgarian midwife to be a significant factor for the optimal course of birth.

$\diamond$ There is a tendency to neglect the integrated approach in the implementation of obstetric care at the expense of instrumental care.

$\diamond$ The psycho-emotional support, provided by the midwife, is essential for optimizing the overall process of providing health care.

$\diamond$ The evaluation of patients is a reliable indicator of the quality of health care.

$\diamond$ The quality of the obstetric care is determined not only by the technological /manipulative/ part of the process. Of great importance for patients is also the attitude of the midwife staff, expressed in empathy, respect for the individual, understanding the emotional state, and support.

\section{REFERENCES}

1. Atanasova Z, Miteva Kr. Developing communication skills in „Health care" students education. Health care. 2009; 1: 21-27.

2. Regulation № 1 for the professional activities that nurses, midwives, associated medical specialists and healthcare assistants may perform according to the instructions or by themselves. MH, 2011.

3. Regulation № 19 for approval of a medical standard „Obstetrics and Gynecology“, ed. MH, SG. 106 /

12.23.2014. 